\title{
Erratum to: Bacterially Expressed Double-Stranded RNAs against Hot-Spot Sequences of Tobacco Mosaic Virus or Potato Virus Y Genome Have Different Ability to Protect Tobacco from Viral Infection
}

\author{
Guo-Hua Yin • Zhao-Nan Sun • Yun-Zhi Song • \\ Hai-Long An • Chang-Xiang Zhu $\cdot$ Fu-Jiang Wen
}

Published online: 8 June 2010

(C) Springer Science+Business Media, LLC 2010

\section{Erratum to: Appl Biochem Biotechnol DOI 10.1007/s12010-010-8968-2}

In the original publication, the order of authorship was incorrect. The correct order is GuoHua Yin, Zhao-Nan Sun, Yun-Zhi Song, Hai-Long An, Chang-Xiang Zhu, and Fu-Jiang Wen. Guo-Hua Yin and Zhao-Nan Sun contributed equally to the paper.

The online version of the original article can be found at http://dx.doi.org/10.1007/s12010-010-8968-2.

G.-H. Yin ·Z.-N. Sun • Y.-Z. Song $\cdot$ H.-L. An • C.-X. Zhu $(\bowtie) \cdot$ F.-J. Wen $(\bowtie)$

College of Life Sciences, State Key Laboratory of Crop Biology, Shandong Agricultural University, Tai'an, Shandong 271018, China

e-mail: zhchx@sdau.edu.cn

e-mail: fjwen@sdau.edu.cn 